INPLASY

PROTOCOL

To cite: Xie et al. Serum Creactive protein as a biomarker in the Ischemic stroke: A metaanalysis. Inplasy protocol 2021110004. doi:

10.37766/inplasy2021.11.0004

Received: 02 November 2021

Published: 02 November 2021

Corresponding author:

Jinlu Xie

02594@zjhu.edu.cn

Author Affiliation:

Huzhou University.

Support: NSFGP.

Review Stage at time of this submission: The review has not yet started.

Conflicts of interest:

None declared.

\section{Serum C-reactive protein as a biomarker in the Ischemic stroke: A meta-analysis}

Review question / Objective: This study aims to explore whether elevated C-reactive protein level is associated with the risk or mortality of Ischemic stroke.

Condition being studied: Ischemic stroke (IS) is a devastating disease causing significant neurologic disability. It is the second leading cause of death and a major cause of longterm disability worldwide. IS is caused by transient or permanent occlusion of cerebral vessels, resulting in cellular damage in the brain and neurologic disability. Neurologic disability, including difficulties with memory, decreased reflexes, cognitive impairment, and aphasia, can reduce quality of life. The identification of predictive biomarkers would contribute to the risk prediction and mortality in IS.

INPLASY registration number: This protocol was registered with the International Platform of Registered Systematic Review and Meta-Analysis Protocols (INPLASY) on 02 November 2021 and was last updated on 02 November 2021 (registration number INPLASY2021110004).

\section{INTRODUCTION}

Review question / Objective: This study aims to explore whether elevated Creactive protein level is associated with the risk or mortality of Ischemic stroke.

Condition being studied: Ischemic stroke (IS) is a devastating disease causing significant neurologic disability. It is the second leading cause of death and a major cause of long-term disability worldwide. IS is caused by transient or permanent occlusion of cerebral vessels, resulting in cellular damage in the brain and neurologic disability. Neurologic disability, including difficulties with memory, decreased reflexes, cognitive impairment, and 
aphasia, can reduce quality of life. The identification of predictive biomarkers would contribute to the risk prediction and mortality in IS.

\section{METHODS}

Participant or population: (1) published in peer-reviewed journals; (2) Englishlanguage literature; (3) reported risk prediction of Ischemic stroke or mortality conferred by CRP levels (4) participants in the general population.

Intervention: Physical exercise was the main intervention (e.g. aerobic exercise, resistance exercise and multicomponent exercise program).

\section{Comparator: Low CRP level group.}

Study designs to be included: Randomized controlled trials (RCTs) will be included.

Eligibility criteria: 1) published in peerreviewed journals; (2) English-language literature; (3) reported risk prediction of Ischemic stroke or mortality conferred by CRP levels (4) participants in the general population.

Information sources: We will search articles in two electronic database including PubMed, and Web of Science. All the English publications until 31 October 2021 will be searched without any restriction of countries or article type. Reference list of all selected articles will independently screened to identify additional studies left out in the initial search.

Main outcome(s): Relative Risk $(95 \%$ Cl) and heterogeneity.

Quality assessment / Risk of bias analysis: A methodological quality assessment of the risks of bias for each study will be analysed.

Strategy of data synthesis: Statistical analysis was performed using Review Manager (Revman Version 5.3., the Cochrane Collaboration, Oxford, UK). The selection of assessments was extracted on study size, sample size, Relative Risk (95\% $\mathrm{Cl})$, and heterogeneity.

Subgroup analysis: Statistical analysis was performed using Review Manager (Revman Version 5.3., the Cochrane Collaboration, Oxford, UK). The selection of assessments was extracted on study size, sample size, Relative Risk $(95 \% \mathrm{Cl})$, and heterogeneity.

Sensitivity analysis: Change the inclusion criteria (especially controversial studies), exclude low-quality studies, and adopt different statistical methods.

Country(ies) involved: China.

Keywords: Ischemic stroke, C-reactive protein, Meta-analysis, Inflammation factors, Biomarker.

Contributions of each author:

Author 1 - Jinlu Xie.

Author 2 - Tao Li.

Author 3 - Tingting He.

Author 4 - Hongwei Yu.

Author 5 - Fangcheng Fan.

Author 6 - Shuaibing He. 